\title{
Valorisation des résidus industriels de pêches pour la transformation de chitosane par technique hydrothermo-chimique
}

\section{The valorization of industrial fishery waste through the hydrothermal-chemical transformation of chitosan}

\author{
To Oanh Truong, Robert Hausler, Fréderic Monette et Patrick Niquette
}

Volume 20, numéro 3, 2007

URI : https://id.erudit.org/iderudit/016170ar

DOI : https://doi.org/10.7202/016170ar

\section{Aller au sommaire du numéro}

\section{Éditeur(s)}

Université du Québec - INRS-Eau, Terre et Environnement (INRS-ETE)

ISSN

1718-8598 (numérique)

Découvrir la revue

Citer cet article

Truong, T. O., Hausler, R., Monette, F. \& Niquette, P. (2007). Valorisation des résidus industriels de pêches pour la transformation de chitosane par technique hydrothermo-chimique. Revue des sciences de l'eau / Journal of Water Science, 20(3), 253-262. https://doi.org/10.7202/016170ar

\section{Résumé de l'article}

Le chitosane est une substance biodégradable d'origine naturelle obtenue par la désacétylation de la chitine, qui se trouve dans l'exosquelette des crustacés. Une de leurs applications plus récentes est le traitement des eaux. Pour favoriser l'utilisation du chitosane des exosquelettes de crustacés (crevettes, crabes, etc.) dans le traitement des eaux usées, les procédés de transformation du chitosane ont été simplifiés et optimisés. Dans la présente étude, les crustacés de crevette grise (Palaemodiae) ont été utilisés pour extraire le chitosane. Les expériences ont porté sur les effets des interactions de la concentration de la solution alcaline, du temps et de la température de réaction sur le degré de désacétylation. En plus de simplifier le processus de transformation de trois à deux étapes, les résultats montrent l'influence de la concentration d'hydroxyde de sodium (7,5-12,5 $\mathrm{M}$ ), du temps de réaction (30 - $180 \mathrm{~min})$ et de la température $\left(80-120^{\circ} \mathrm{C}\right)$ sur le degré de désacétylation. La méthode de la spectroscopie infrarouge en film mince a été utilisée pour analyser le degré de désacétylation du chitosane. Un degré de désacétylation de $90 \%$ a été atteint avec une concentration de la solution alcaline de $12,5 \mathrm{M}$, un temps de réaction de 120 min et une température de $110^{\circ} \mathrm{C}$. Par ailleurs, en réduisant le nombre d'étapes de transformation et la consommation de réactifs, le procédé développé est plus économique et a une meilleure performance environnementale. 


\title{
VALORISATION DES RÉSIDUS INDUSTRIELS DE PÊCHES POUR LA TRANSFORMATION DE CHITOSANE PAR TECHNIQUE HYDROTHERMO-CHIMIQUE
}

\author{
The valorization of industrial fishery waste through the hydrothermal-chemical transformation of chitosan
}

To Oanh Truong ${ }^{1}$, Robert Hausler ${ }^{2 *}$, Fréderic Monette ${ }^{2}$ et Patrick Niquette ${ }^{2}$

${ }^{1}$ Institut des sciences de l'environnement, Université du Québec à Montréal (UQÀM), C.P. 8888, succursale Centre-Ville, Montréal (Québec), Canada, H3C 3P8

${ }^{2}$ Station expérimentale des procédés pilotes en environnement, École de technologie supérieure (ÉTS), Université du Québec, 1100, rue Notre-Dame Ouest, Montréal, (Québec), Canada, H3C 1K3

Reçu le 6 janvier 2005, accepté le 6 juin 2006

\section{RÉSUMÉ}

Le chitosane est une substance biodégradable d'origine naturelle obtenue par la désacétylation de la chitine, qui se trouve dans l'exosquelette des crustacés. Une de leurs applications plus récentes est le traitement des eaux. Pour favoriser l'utilisation du chitosane des exosquelettes de crustacés (crevettes, crabes, etc.) dans le traitement des eaux usées, les procédés de transformation du chitosane ont été simplifiés et optimisés. Dans la présente étude, les crustacés de crevette grise (Palaemodiae) ont été utilisés pour extraire le chitosane. Les expériences ont porté sur les effets des interactions de la concentration de la solution alcaline, du temps et de la température de réaction sur le degré de désacétylation. En plus de simplifier le processus de transformation de trois à deux étapes, les résultats montrent l'influence de la concentration d'hydroxyde de sodium $(7,5-12,5 \mathrm{M})$, du temps de réaction $(30-180 \mathrm{~min})$ et de la température $\left(80-120^{\circ} \mathrm{C}\right)$ sur le degré de désacétylation. La méthode de la spectroscopie infrarouge en film mince a été utilisée pour analyser le degré de désacétylation du chitosane. Un degré de désacétylation de $90 \%$ a été atteint avec une concentration de la solution alcaline de $12,5 \mathrm{M}$, un temps de réaction de $120 \mathrm{~min}$ et une température de $110^{\circ} \mathrm{C}$. Par ailleurs, en réduisant le nombre d'étapes de transformation et la consommation de réactifs, le procédé développé est plus économique et a une meilleure performance environnementale.

Mots clés : Exosquelettes/carapaces de crevettes, Chitosane, $N$-désacétylation, Chitine, Hydrothermo-chimique, Valorisation.

\section{SUMMARY}

Chitosan is a natural biodegradable biopolymer produced from chitin, a polysaccharide derived from the shells of shrimp, crab and lobster. The development of commercial applications of chitin and chitosan in different fields such as biomedicine, nutrition, food processing, agriculture, cosmetics, and wastewater treatment has rapidly expanded in recent years. This paper investigates the hydrothermal production of 
chitosan from the carapace of gray shrimp (Palaemodiae) for use as a coagulant in wastewater treatment.

To obtain chitosan from shrimp exoskeletons, they were treated following two different steps. The first step was the demineralization of the shrimp's exoskeleton, where calcium was removed using dilute $\mathrm{HCl}$. The second deacetylation step completely dissolved the shrimp exoskeleton using a $\mathrm{NaOH}$ solution. In both steps, the ratio between solution digestive and exoskeleton was studied (1:10 (w:v)). For the demineralization process, the concentration of $\mathrm{HCl}$ was varied from 0.5 to $3.5 \mathrm{M}$ in $0.5 \mathrm{M}$ intervals at constant temperatures of $25^{\circ} \mathrm{C}$ and $50^{\circ} \mathrm{C}$. The comparison of the results using these two temperatures indicated that the most favourable demineralization occurred after $6 \mathrm{~h}$ at $25^{\circ} \mathrm{C}$ and after $2 \mathrm{~h}$ at $50^{\circ} \mathrm{C}$ at a $\mathrm{HCl}$ concentration of $2 \mathrm{M}$. In this case, it was not necessary to use a $\mathrm{HCl}$ concentration greater than $2 \mathrm{M}$ due to the fixed reaction time. When the $\mathrm{HCl}$ solution was heated, the reaction time of the demineralization process was reduced by a factor of three compared to that when room temperature $\mathrm{HCl}$ was used under the same conditions. Moreover, this reaction followed a pseudo-second-order equation with approximate rate constant of $2.38 \mathrm{~L} \mathrm{~g}^{-1} \mathrm{~min}^{-1}$ at $25^{\circ} \mathrm{C}$ in $1.5 \mathrm{M} \mathrm{HCl}$.

The effectiveness of the transformation to chitosan depends on the interaction among the sodium hydroxide concentration, the reaction time and the temperature at which the deacetylation process occurs. The influence of the concentration of the alkaline solution, the reaction temperature, and the reaction time on the degree of deacetylation (DD) was investigated. The DD obtained was quantitatively analyzed by thin film infrared spectroscopy (IR). Film thickness was measured by using a micrometer with the smallest possible unit measurement count of $0.01 \mathrm{~mm}$. First, exoskeletons were exposed to $\mathrm{NaOH}$ concentrations of $7.5 \mathrm{M}, 10 \mathrm{M}$ and $12.5 \mathrm{M}$ for $60 \mathrm{~min}$ at various temperatures ranging from $60^{\circ} \mathrm{C}$ to $120^{\circ} \mathrm{C}$ at intervals of $20^{\circ} \mathrm{C}$. Second, the reaction time was changed from 30 to $180 \mathrm{~min}$ at $100^{\circ} \mathrm{C}$ at $30 \mathrm{~min}$ intervals using the same $\mathrm{NaOH}$ concentrations previously mentioned. The results show that the factors that influence the $\mathrm{DD}$ values were the reaction temperature and the concentration of $\mathrm{NaOH}$. However, based on these experiments, the concentration of $\mathrm{NaOH}$ influences the DD values the most. The DD values of chitosan production began to reach a constant level when the reaction temperature was greater than $100^{\circ} \mathrm{C}$. As a result, after one hour at $110^{\circ} \mathrm{C}$, chitosan production was obtained with different DD values of $60 \%, 67 \%$, and $78 \%$ at $\mathrm{NaOH}$ concentrations of $10 \mathrm{M}, 11.25 \mathrm{M}$ and $12.5 \mathrm{M}$ respectively. Nevertheless, chitosan could not be formed at $7.5 \mathrm{M} \mathrm{NaOH}$, even though the reaction time was $3 \mathrm{~h}$. Furthermore, when the reaction time was longer than $120 \mathrm{~min}$, the $\mathrm{DD}$ values of chitosan increased slowly.

Therefore, the optimum conditions required for the deacetylation production of chitosan from gray shrimp carapace (DD of 90\%), to be applied in wastewater treatment, are as follows: the exoskeleton should be exposed to a solution of $12.5 \mathrm{M} \mathrm{NaOH}(45 \%)$ for $2 \mathrm{~h}$ at $110^{\circ} \mathrm{C}$. This product is generally termed chitosan when it has greater than $65 \%$ of the acetylic groups removed.

By diminishing the number of steps and reducing the chemical reagents needed, this study demonstrates the economical and environmental advantages of using chitosan as a coagulant to treat wastewater.

\section{Key words: Shrimp exoskeleton, Carapace, Chitosan, N-deacetylation, Chitin, Hydrothermal-chemistry, Valori- zation.}

\section{INTRODUCTION}

La découverte de la chitine et du chitosane date du $18^{\mathrm{e}}$ siècle, mais ce n'est que dans les années 1970 que ces deux produits ont suscité un réel intérêt (MUZZARELLI, 1977; ROBERTS, 1992). La chitine et le chitosane sont des produits des transformations successives des exosquelettes (carapaces) de crustacés provenant des industries agro-alimentaires. Plus spécifiquement, le chitosane est obtenu par N-désacétylation de la chitine (Figure 1).

Le chitosane est un biopolymère polycationique non toxique, biodégradable et biocompatible, soluble dans les acides organiques dilués sous forme de gel chargé positivement (BRINE et al., 1992; CUROTTE et AROS, 1993; GOOSEN, 1997; JUANG et SHIAU, 2000). Le chitosane a donc des propriétés chimiques et biologiques utilisables dans de nombreuses applications industrielles, médicales et environnementales (AIBA et al., 1985; DAUTZENBERG et

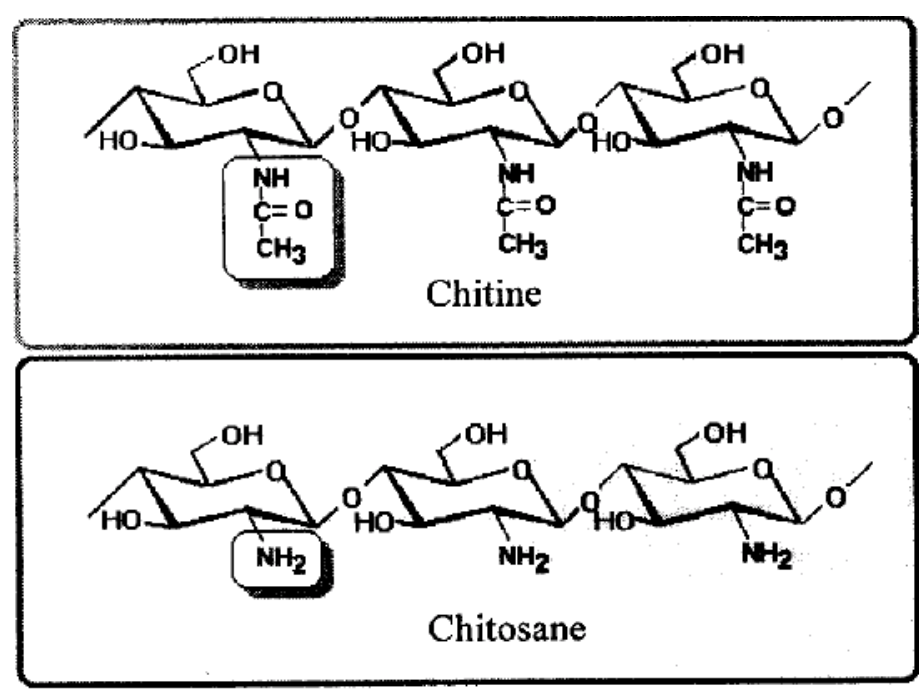

Figure 1. Structure chimique de la chitine et du chitosane. Chemical structure of chitin and chitosan. 
al., 1994; HIRANO, 1989; MUZZARELLI, 1996). Plusieurs études ont montré que le chitosane peut être utilisé comme coagulant/floculant cationique naturel pour le traitement des eaux usées (CHEN et al., 2003; COUGHLIN et al., 1990; HIRANO, 1996, 1999; KIM et al., 1999; MURCOTTE et HARLEMAN, 1993a, b).

La production de chitosane est décrite à la figure 2 et, fondamentalement, elle comprend trois étapes successives (déminéralisation, déprotéination et désacétylation). Pour réaliser l'étape de désacétylation, trois voies différentes ont été développées.

La première voie, appelée la méthode chimique, utilise uniquement du $\mathrm{NaOH}$ concentré et requiert entre trois à quatre jours pour atteindre un degré de désacétylation de 70 à $75 \%$ (HIRANO, 1989). La seconde voie repose sur une réaction enzymatique faisant appel aux enzymes trypsine, chitosanase et/ou mycelia (FREHAUT, 2002; NWE et STEVENS, 2002). Les hydrolyses enzymatiques sont effectuées en $28 \mathrm{~h}$, pour atteindre le même de degré désacétylation, 74 \% (PELLETIER et al., 1990). Les inconvénients majeurs de cette voie sont les quantités importantes d'enzymes nécessaires à la réaction (691 mg-chitosanase/g-chitine) et la difficulté de les isoler (PLONSKI et al., 1990). La troisième voie de désacétylation est réalisée par une technique thermomécanique en utilisant des autoclaves en cascade. Cette technique nécessite un milieu basique $\mathrm{NaOH}$, une température de $230^{\circ} \mathrm{C}$ et une pression de 17,24 MPa (2 500 psi). Elle semble être la plus rapide des trois voies puisqu'elle nécessite $24 \mathrm{~h}$ pour atteindre un degré de désacétylation de $71 \%$ (CHATTERJEE et al., 2005; NIOLA, 1991). L'inconvénient majeur de cette technique réside dans

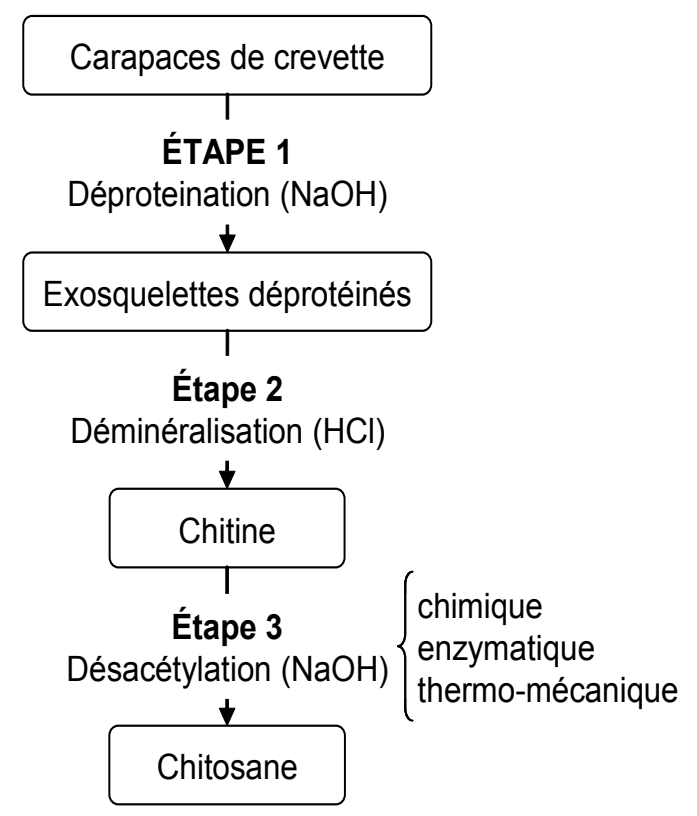

Figure 2. Étapes de l'extraction du chitosane des exosquelettes de crevettes (en trois étapes). Extraction of chitosan from shrimp exoskeletons (in three steps). les besoins d'infrastructures tels que l'installation d'autoclaves en cascade avec une pressurisation sous atmosphère d'azote (NO et al., 2000; PELLETIER, 1991).

Dans ce contexte, et pour favoriser une utilisation environnementale du chitosane, le processus de transformation des exosquelettes de crustacé doit être simplifié en vue de réduire les coûts de production. Cette publication présente donc les résultats des expériences réalisées afin d'atteindre cet objectif et, plus particulièrement, l'étude des paramètres techniques de l'extraction, ainsi que les concentrations d'acide et de base.

\section{MATÉRIELS ET MÉTHODES}

Les exosquelettes (carapaces) de crevettes grises (Palaemodiae) fraîches ont été utilisés dans cette étude pour extraire le chitosane. Tous les réactifs utilisés dans la procédure d'extraction du chitosane des exosquelettes de crevettes sont de grade ACS.

\subsection{Procédure}

Le chitosane a été obtenu après les deux étapes suivantes : une déminéralisation en milieu acide (étape 1) suivie d'une déprotéination et d'une désacétylation simultanée par voie hydrothermo-chimique en milieu basique (étape 2). Lors des procédures d'extraction du chitosane, différentes concentrations de solutions digestives d'acide $\mathrm{HCl}$ et de base $\mathrm{NaOH}$, variant respectivement de 0,3 à $3,5 \mathrm{M}$ et de 7,5 à $12,5 \mathrm{M}$, ont été utilisées.

\subsection{1 Étape 1-Déminéralisation}

Les carapaces, une fois prétraitées (lavage, broyage et séchage), ont été minutieusement mélangées dans des solutions aqueuses d'acide chlorhydrique $(\mathrm{HCl})$. Tout au long de l'expérience, le rapport de solide-liquide est de 1:10 p/v (poids de carapace sec/volume de solution $\mathrm{HCl}$ dilué). Dans les essais réalisés à température ambiante $\left(25^{\circ} \mathrm{C}\right)$, les échantillons ont été mélangés dans une gamme de concentration d'acide $\mathrm{HCl}$ allant de 0,3 à 3,5 M. En parallèle, des essais de déminéralisation à $50{ }^{\circ} \mathrm{C}$ ont été réalisés avec de l'acide $\mathrm{HCl}(\mathrm{de} 0,3$ à $3 \mathrm{M}$ ) grâce à une plaque chauffante électrique.

\subsection{2 Étape 2 - Protéination et désacétylation}

Ces deux étapes ont été effectuées simultanément en milieu alcalin. Les carapaces déminéralisées, après des lavages à l'eau jusqu'au $\mathrm{pH}$ neutre, ont été mélangées dans des solutions de soude caustique $(\mathrm{NaOH})$ en proportion $1: 10 \mathrm{p} / \mathrm{v}$ (poids de carapace sec/volume $\mathrm{NaOH}$ ). Les expériences de cette 
étape ont été réalisées avec des concentrations de $\mathrm{NaOH}$ de $7,5,10$ et $12,5 \mathrm{M}$, à des températures de 80,100 et $120^{\circ} \mathrm{C}$ pendant des périodes allant de 30 à $180 \mathrm{~min}$. Durant le traitement simultané de déprotéination-désacétylation, des échantillons insolubles ont été prélevés toutes les 30 min à des fins d'analyses.

\subsection{Analyses}

Les échantillons prélevés lors de la déminéralisation ont subi une précipitation à l'oxalate pour analyser la décalcification de manière qualitative (GASTON, 1983) et analyser quantitativement par réaction de complexation avec de l'EDTA (APHA et al., 1995) afin de contrôler le processus de la réduction de sel de calcium.

Pour l'étape de déprotéination et désacétylation, les carapaces traitées ont été examinées par la réaction oxydoréductrice avec une solution $\mathrm{I}_{2} / \mathrm{K}_{2} \mathrm{CrO}_{4}$ en milieu acide et/ou par la solubilité dans l'acide $\mathrm{AcOH} 2 \%$ afin d'évaluer la qualité (apparente) du produit chitosane extrait (HIRANO, 1989; MUZZARELLI et ROCHETTI, 1985; NO et al., 2000).

Le degré des groupes $-\mathrm{NH}_{2}$ libres dans la molécule du chitosane (degré de désacétylation) a été déterminé par spectroscopie infra-rouge (BLAIR et al., 1989; MOORE et ROBERTS, 1980; WANG et al., 2004). Les échantillons du chitosane obtenus ont été disposés en films minces. Après avoir au préalable préparé la solution du chitosane $1 \%$ (poids du chitosane/volume de $\mathrm{AcOH}$ ) dans l'acide acétique $1 \%(\mathrm{v} / \mathrm{v})$, celui-ci a été versé au compte goutte sur un pétrie et séché dans l'étuve à $60^{\circ} \mathrm{C}$ pendant $12 \mathrm{~h}$. Les films se sont développés sous forme $\mathrm{NH}_{3}^{+}$, ils doivent être déprotonés par lavages en trois étapes, de la manière suivante : d'abord avec une solution d'un mélange méthanol pur et ammoniaque 0,4 M (9:1 de v/v) en plusieurs fois, puis avec l'eau distillée, et finalement, avec le méthanol. Les films du chitosane obtenus ont été conservés à $80^{\circ} \mathrm{C}$ pendant $16 \mathrm{~h}$ avant de relever les spectres infra-rouge (KHAN et al., 2002). Ces spectres ont été effectués à une longueur d'onde comprise entre 400 à $4000 \mathrm{~cm}^{-1}$ en utilisant un spectromètre 5-DBX avec une résolution de $2 \mathrm{~cm}^{-1}$ (CHEN et al., 2004).

Cette méthode de caractérisation du chitosane par IR est basée sur la relation entre la valeur de l'absorbance (A) de l'amide primaire à $1655 \mathrm{~cm}^{-1}$ et celle de l'hydroxyle à $3450 \mathrm{~cm}^{-1}$. Le degré de désacétylation (DD) a été calculé par l'équation 1 (BAXTER et al., 1992; KHAN et al., 2002; TOKURA et NISHI, 1994) :

$$
\mathrm{DD} \%=100-\left[\left(\frac{\mathrm{A}_{1655}}{\mathrm{~A}_{3450}}\right) * 115\right]
$$

\section{RÉSULTATS ET DISCUSSION}

\subsection{Influence du temps sur le procédé de déminéralisation en fonction de la concentration d'acide digestive}

La figure 3 présente une étude de la cinétique de la déminéralisation des exosquelettes de crevettes grises. Selon l'étude cinétique de la réaction, la déminéralisation est égale à un pseudo ordre 2 . Par exemple, à $25^{\circ} \mathrm{C}$ pour une concentration de $1,5 \mathrm{M}$ d'HCl, la constante de vitesse est de $1,87 \mathrm{~L} \mathrm{~g}^{-1} \mathrm{~min}^{-1}$. Dans ces conditions, la déminéralisation est complétée en environ $6 \mathrm{~h}$.

L'orde de réaction (ordre 2) s'explique par le fait que la structure des exosquelettes de crustacés comporte plusieurs couches : la couche cornée extérieure, la couche de pigment, la couche de chitine et la couche épidermique (OKAFOR, 1965). L'étape de déminéralisation est une absorption de la solution acide sur les différentes couches carbonatées selon la réaction simplifiée et globale suivante (Équation 2) :

$$
\mathrm{CaCO}_{3}+2 \mathrm{H}^{+} \rightarrow \mathrm{Ca}^{2+}+\mathrm{H}_{2} \mathrm{O}+\mathrm{CO}_{2} \nearrow
$$

Ainsi, plus la concentration d'acide est élevée, plus les couches sont attaquées rapidement par l'absorption de l'acide. D'autre part, l'augmentation de la température accélère non seulement la réaction de déminéralisation proprement dite, mais elle semble permettre également de ramollir la carapace, facilitant du même coup l'absorption de l'acide.

La figure 4 permet de comparer les résultats de la déminéralisation réalisée sous différentes concentrations de $\mathrm{HCl}$ et à deux températures $\left(25\right.$ et $\left.50^{\circ} \mathrm{C}\right)$.

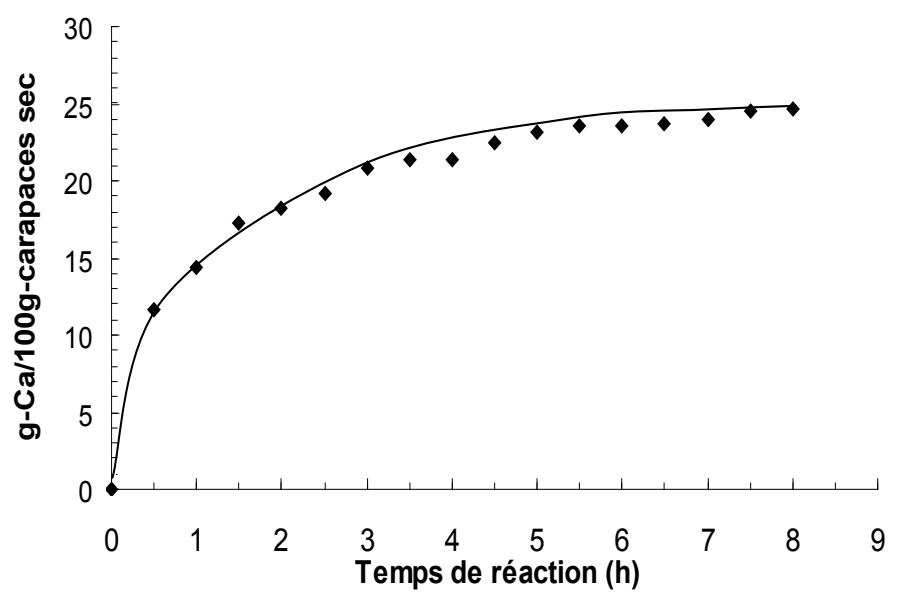

Figure 3. Influence du temps sur la déminéralisation des exosquelettes de crevettes à température ambiante $\left(25^{\circ} \mathrm{C}\right)$ par $\mathrm{HCl} 1,5 \mathrm{M}$. Influence of time on the demineralization process of shrimp exoskeletons at room temperature $\left(25^{\circ} \mathrm{C}\right)$ in $1.5 \mathrm{M} \mathrm{HCl}$. 


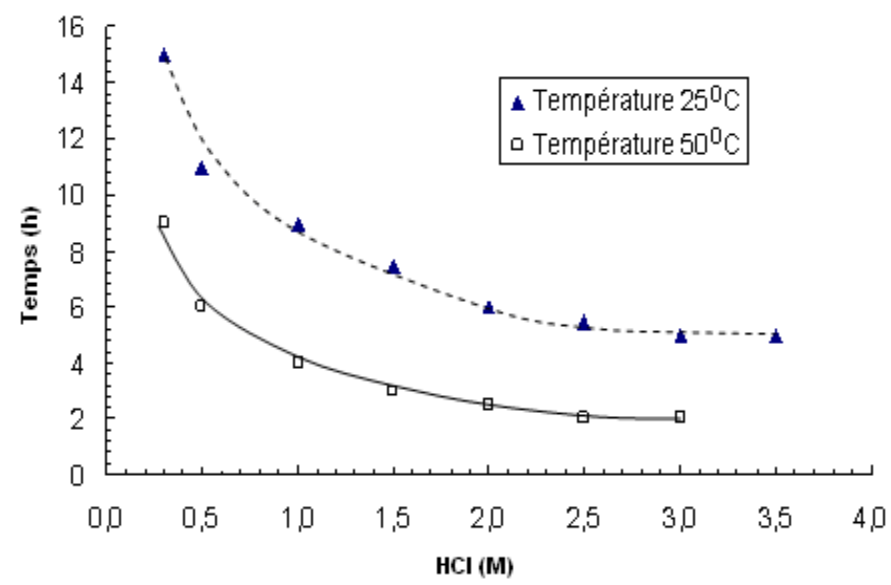

Figure 4. Influence de la concentration d'acide $\mathrm{HCl}$ sur le temps de la déminéralisation des exosquelettes de crevettes à température ambiante $\left(25^{\circ} \mathrm{C}\right)$ et à $50^{\circ} \mathrm{C}$.

Influence of $\mathrm{HCl}$ concentration on the time required for the demineralization of shrimp exoskeletons at room temperature $\left(25^{\circ} \mathrm{C}\right)$ and at $50^{\circ} \mathrm{C}$.

À $25^{\circ} \mathrm{C}$, des concentrations d'HCl élevées $(2,5,3$ et $3,5 \mathrm{M})$ ne semblent plus influencer significativement le temps nécessaire à une déminéralisation. Les exosquelettes de crevettes sont complètement décalcifiés en $6 \mathrm{~h}$ environ. À $50{ }^{\circ} \mathrm{C}$, la déminéralisation est obtenue en moins de $3 \mathrm{~h}$ avec des concentrations d' $\mathrm{HCl}$ respectives de 1,5, 2,0 ou 2,5 M.

Puisque l'élévation de température permet à la fois de réduire de 2 à 3 fois le temps de réaction (infrastructure plus petite) et la quantité d'acide d'environ 2 fois par rapport à $25^{\circ} \mathrm{C}$ (réduction de produits chimiques), les conditions préconisées pour la déminéralisation sont : une température de $50{ }^{\circ} \mathrm{C}$ et une concentration d'acide de $2 \mathrm{M}$. Toutefois, la température de déminéralisation plus élevée $\left(>50^{\circ} \mathrm{C}\right)$ n'est pas avantageuse $\mathrm{du}$ point de vue environnemental. Cette élévation thermique augmenterait le taux d'évaporation. Cela pourrait causer des impacts environnementaux.

\subsection{Influence de la concentration de $\mathrm{NaOH}$ et de la température sur la désacétylation}

Dans le cadre des essais expérimentaux, l'interaction de la concentration de la solution alcaline et de la température a été étudiée pour un temps de réaction constant d'une heure. La réaction de désacétylation en milieu basique est résumée par l'équation suivante (Équation 3) :

$$
\mathrm{R}-\mathrm{NHCOCH}_{3}+\mathrm{OH}^{-} \rightarrow \mathrm{R}-\mathrm{NH}_{2}+\mathrm{CH}_{3} \mathrm{COO}^{-}(3)
$$

Pour la concentration de $\mathrm{NaOH}$ la plus faible $(7,5 \mathrm{M})$, le pourcentage de désacétylation augmente à partir d'environ $60^{\circ} \mathrm{C}$, mais il reste faible même à $120^{\circ} \mathrm{C}$ (Figure 5). Le produit extrait reste majoritairement sous forme du chitine. Par contre, le degré de désacétylation augmente plus rapidement pour les

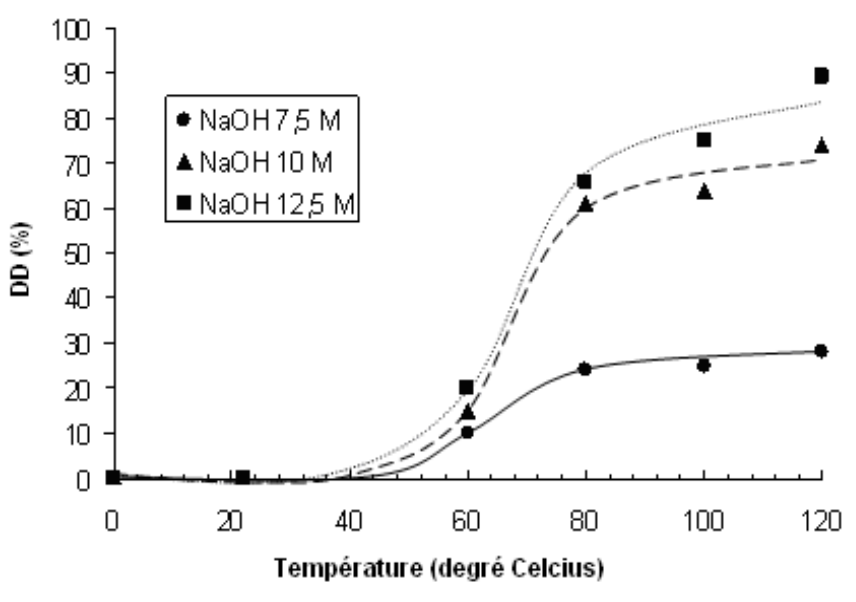

Figure 5. Influence de la température sur le degré de désacétylation après une heure pour différentes concentrations de $\mathrm{NaOH}$. Influence of temperature on the degree of deacetylation at different $\mathrm{NaOH}$ concentrations after one hour.

concentrations de $\mathrm{NaOH}$ plus élevées $(10$ ou $12,5 \mathrm{M})$. Ces résultats vérifient l'hypothèse selon laquelle l'interaction de la concentration et de l'énergie thermique sont les principaux critères à prendre en compte pour la réaction de désacétylation. L'analyse des résultats de la mesure du degré de désacétylation montre que celui-ci a augmenté approximativement de $8 \%(66$ à $74 \%$ de $\mathrm{DD})$, à une concentration élevée de $\mathrm{NaOH}(10 \mathrm{M})$ et avec un accroissement thermique de $20^{\circ} \mathrm{C}\left(100\right.$ à $\left.120^{\circ} \mathrm{C}\right)$. Cette variation de DD semble moins importante, seulement de $+2 \%$ (26 à $28 \%$ de DD) à faible concentration de $\mathrm{NaOH}$ (7,5 M). Cet écart peut être considéré comme erreur d'analyse. Cependant, cet accroissement de DD est particulièrement significatif $(13 \%)$ avec une augmentation de concentration de la base de 2,5 M (10 M à 12,5 M). Ainsi, la variation de concentration de $\mathrm{NaOH}$ influence plus significativement la vitesse de réaction du DD que la température. La température, dans cette étape, est un facteur qui affaiblit la liaison des groupes acétyle et accélère la réaction de désacétylation.

\subsection{Influence de la concentration de $\mathrm{NaOH}$ et du temps sur la désacétylation}

Dans cette réaction de désacétylation, il s'agit d'une interrelation entre les variables suivantes : la concentration de la solution basique, la température et le temps de réaction.

La désacétylation en fonction de la concentration de $\mathrm{NaOH}$ étudiées (de 7,5 à 12,5 M) et du temps de réaction à une température constante de $110^{\circ} \mathrm{C}$ est présentée à la figure 6. Cette réaction de désacétylation semble être complétée après une période variant de 90 à 120 min selon la concentration en $\mathrm{NaOH}$. Par ailleurs, pour une concentration de $\mathrm{NaOH}$ plus faible $(8,75 \mathrm{M})$, cette réaction est incomplète même après $3 \mathrm{~h}$. Pour différents auteurs (CHANG et al., 


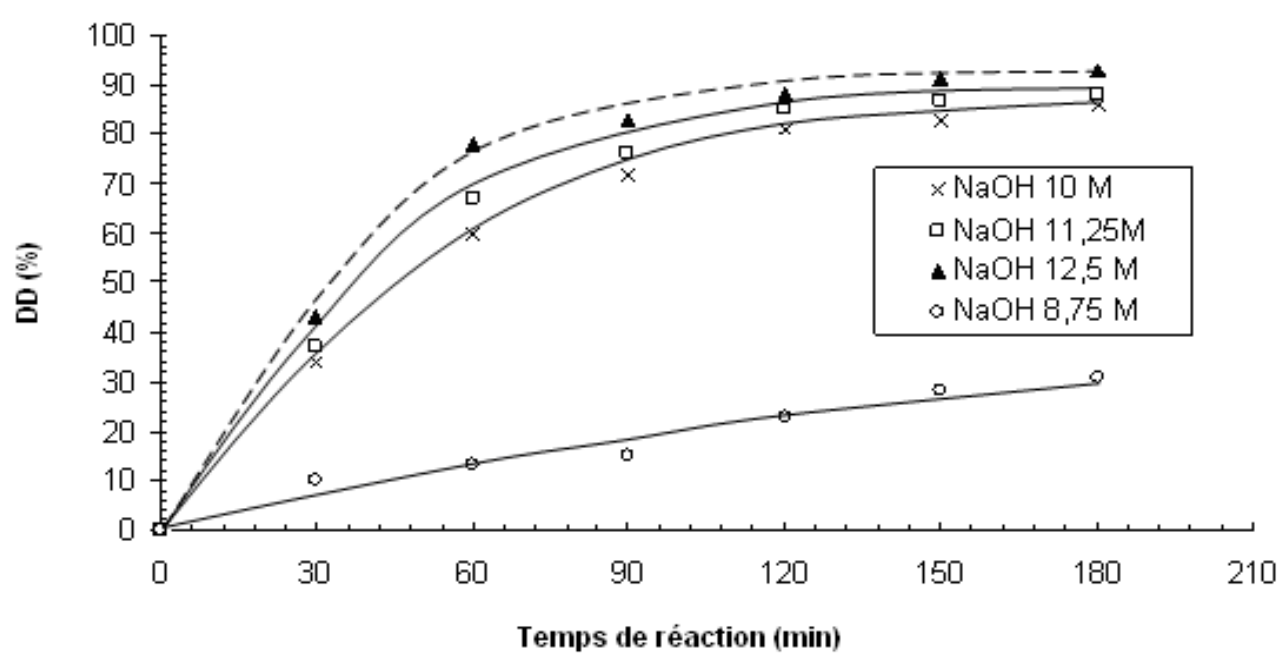

Figure 6. Influence de la concentration de $\mathrm{NaOH}$ et du temps de réaction sur le degré de désacétylation à $110{ }^{\circ} \mathrm{C}$.

Influence of $\mathrm{NaOH}$ concentration and reaction time on the degree of deacetylation at $110^{\circ} \mathrm{C}$.

1997; METHACANON et al., 2003), cette limite peut être expliquée par la cinétique Ea de la réaction de désacétylation. Les liaisons entre les groupes d'amine et d'acétyle n'ont pas été complètement coupées en raison de l'énergie d'activation qui n'est pas suffisante dans ce cas. Par conséquent, dans le cas d'une faible concentration de $\mathrm{NaOH}$ (inférieure à $10 \mathrm{M}$ ), la désacétylation ne se produit pas complètement. Les produits de désacétylation, dans ces conditions expérimentales, sont représentatifs de la composition mixte chitine/chitosane. Ainsi, pour obtenir un chitosane de bonne qualité, il faut reprendre le traitement plusieurs fois à l'aide de solution $\mathrm{NaOH}$ (FENTON et EVELEIGH, 1981; WU, 1988). Ce régime de retraitement n'est techniquement pas facile et économiquement peu rentable à grande échelle.

L'analyse des résultats (Figure 6) montre que la meilleure désacétylation a commencé après $60 \mathrm{~min}$, pour les trois concentrations de $\mathrm{NaOH}$ étudiées. À ce moment, l'énergie d'activation pour la désacétylation peut être atteinte et la rupture de liaisons chimiques a lieu. Toutefois, cette rupture des groupements d'acétyle n'est significative qu'au niveau de la concentration alcaline élevée (>10 M). La diffusion de la solution alcaline sur le substrat de carapaces augmente en fonction de temps. Ces résultats obtenus (Figure 6) confirment l'hypothèse qu'il existe une interaction entre la concentration de $\mathrm{NaOH}$ et le temps de réaction sur le DD.

\section{4 Évaluation des conditions optimales d'extraction duchitosane des exosquelettes de crevettes grises (Palaemodiae)}

Dans la présente étude, la valorisation des exosquelettes de crevettes en chitosane a été réalisée par la technique hydrothermo-chimique en deux étapes. Dans la première étape, la déminéralisation se réalise à une température de $50{ }^{\circ} \mathrm{C}$ et une concentration d' $\mathrm{HCl}$ de 1,5 M, le temps de déminéralisation a été réduit de deux à trois fois relativement au traitement sans chauffage, ce qui est plus économique (Tableau 1).

Dans la deuxième étape, les trois facteurs influençant la désacétylation sont : la concentration en $\mathrm{NaOH}$, la température et le temps de réaction. Toutefois, le degré de désacétylation dépend aussi des conditions de l'étape précédente: la déminéralisation et le prétraitement. La désacétylation en milieu alcalin est difficile si la déminéralisation n'est pas complète et la qualité du chitosane extrait semble moins bonne. D'un autre côté, la concentration de $\mathrm{NaOH}$ influence de façon importante la variation de la température de réaction. METHACANON

Tableau 1. Conditions optimales de l'extraction du chitosane en deux étapes.

Table 1. Optimal conditions for chitosan extraction in two steps.

\begin{tabular}{|c|c|c|c|c|}
\hline Étape & Température $\left({ }^{\circ} \mathrm{C}\right)$ & Solution digestive & Temps (h) & DD (\%) \\
\hline Déminéralisation & 50 & $\mathrm{HCl} 2 \mathrm{M}$ & 2,5 & \\
\hline $\begin{array}{l}\text { Déprotéination et } \\
\text { désacétylation }\end{array}$ & 110 & $\mathrm{NaOH} 12,5 \mathrm{M}$ & 2,0 & 90 \\
\hline
\end{tabular}


et al. (2003) mentionnent qu'à une concentration de $\mathrm{NaOH}$ de 10 à $15 \mathrm{M}$, l'activation énergétique Ea de la désacétylation est, respectivement, d'environ de 22 à $50 \mathrm{~kJ} / \mathrm{mol}$, ce qui permet d'augmenter le degré de désacétylation.

La vitesse de la désacétylation dépend donc non seulement de la qualité de $\mathrm{NaOH}$ utilisé mais aussi de la température pour une rupture des liaisons d'acétyle. Toutefois, une température élevée entraîne une dégradation du chitosane qui fait chuter la viscosité $\eta$ et diminuer la masse moléculaire $M$ $(\eta=\mathrm{kM}$ a), ce qui affecte la solubilité du chitosane. La variation de la concentration de $\mathrm{NaOH}$, elle, n'affecte pas sa masse moléculaire (METHACANON et al., 2003). En conséquence, la production de chitosane à partir des exosquelettes de crevettes à une température plus haute n'est pas fiable. De plus, la production du chitosane à haute température, qui consiste à fusionner la chitine, entraîne une dégradation du contenant et implique donc des manipulations plus difficiles à adapter pour l'industrie. Pour s'assurer de la qualité du chitosane produit, la température de digestion doit être maintenue constante à $110^{\circ} \mathrm{C}$.

La production de chitosane, à partir d'exosquelettes de crevettes séchées, peut être réalisée en un jour au lieu des trois jours nécessaires par la méthode conventionnelle. Les résultats obtenus montrent que le chitosane extrait a un DD supérieur à $90 \%$ dans des conditions optimales par la technique hydrothermo-chimique en deux étapes à $110^{\circ} \mathrm{C}$, soit : $11,25 \mathrm{M}$ de $\mathrm{NaOH}$ pendant $3 \mathrm{~h}$ ou $12,5 \mathrm{M} \mathrm{NaOH}$ durant $2 \mathrm{~h}$.

Pour l'extraction du chitosane à un DD de $85 \%$ dans le cadre du traitement des eaux, la procédure de transformation doit être réalisée aux conditions optimales suivantes :

- Déminéralisation à $50^{\circ} \mathrm{C}$ durant $2,5 \mathrm{~h}$ dans $\mathrm{HCl} 2 \mathrm{M}$;

- Déprotéination et désacétylation à $110^{\circ} \mathrm{C}$ par $\mathrm{NaOH}$ $11,25 \mathrm{M}$ durant $2 \mathrm{~h}$.
D'un autre côté, cette interprétation a été examinée par le plan factoriel d'expériences afin de modéliser et d'optimiser les conditions opératoires pour la production du chitosane. La concentration de $\mathrm{NaOH}$, la température et le temps de réaction ont été choisis comme paramètres indépendants pour le plan factoriel à trois variables et à trois niveaux (maximum, moyen et minimum) (Tableau 2).

Les résultats obtenus dans diverses conditions ont été analysés statistiquement en utilisant une analyse de régression multilinéaire (valeur de l'incertitude $=0,05$ ). La meilleure équation obtenue pour le processus d'extraction du chitosane dans cette étude, est la suivante (Équation 4):

$$
\begin{aligned}
& \% \mathrm{DD}=72,56+5,6^{*}(\mathrm{NaOH})+3,78^{*}(\text { température }) \\
& +24,86 *(\text { temps })-11,92^{*}(\text { temps })^{2}
\end{aligned}
$$

Les valeurs de cette équation indiquent que l'effet de trois facteurs étudiés est sensible sur le DD en ordre : temps de réaction $(24,86)>$ concentration $\mathrm{NaOH}(5,6)>$ température réactionnelle $(3,78)$. La surface de réponse de variation du $\mathrm{DD}$ en fonction de la concentration de $\mathrm{NaOH}$ et en fonction du temps est présentée à la figure 7 . En outre, un temps de réaction de $152 \mathrm{~min}(\approx 2,5 \mathrm{~h})$ est prédit à partir de l'équation 4 pour atteindre $90 \%$ de DD en utilisant une concentration de $\mathrm{NaOH}$ de $12,5 \mathrm{M}$ et une température fixée à $110^{\circ} \mathrm{C}$. Ce temps prédit par le plan factoriel semble conforme au temps réel de réaction de désacétylation $(120 \mathrm{~min})$ avec une erreur de $27 \%$.

Basée sur les résultats obtenus, la production du chitosane par la technique hydrothermo-chimique en deux étapes est plus simple et s'avère prometteuse par rapport aux méthodes couramment utilisées. En effet, le temps de réaction est plus court (un jour), la quantité de réactifs et l'énergie nécessaires sont moins importantes que les techniques classiques. Pour ces raisons, cette technique permet de réduire les coûts de production et représente un intérêt pour le secteur de la

Tableau 2. Conditions opératoires de l'extraction du chitosane (plan d'expériences du type 33).

Table 2. Operating conditions for chitosan extraction: Factorial experimental design.

\begin{tabular}{ccccc}
\hline Paramètre & Paramètre codé* & Valeur codée & maximale (+1)* \\
\cline { 3 - 5 } Concentration & $\mathrm{x}_{1}$ & minimale (-1)* & moyenne (0)* & 12,5 \\
de $\mathrm{NaOH}(\mathrm{M})$ & $\mathrm{x}_{2}$ & 10 & 11,25 & 110 \\
Température $\left({ }^{\circ} \mathrm{C}\right)$ & $\mathrm{x}_{3}$ & 30 & 90 & 150 \\
Temps $(\mathrm{min})$ & 30 & & 100 \\
\hline
\end{tabular}

*Code du paramètre: valeur adimensionnelle rendant homogène le modèle mathématique. 


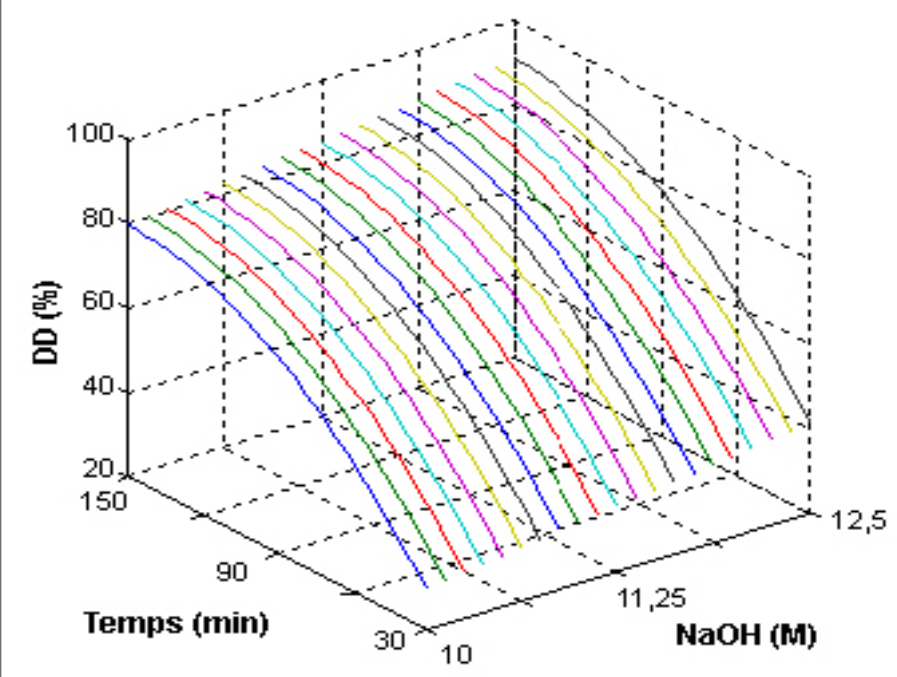

Figure 7. Effet de la concentration du $\mathrm{NaOH}$ et du temps sur le DD du chitosane des carapaces de crevettes grises (Palaemodiae). Effect of $\mathrm{NaOH}$ concentration and reaction time on the degree of deacetylation of gray shrimp (Palaemodiae) chitosan.

transformation des résidus de la pêche industrielle sur le plan de l'extraction du chitosane.

La valorisation des exosquelettes de crevettes, par la voie hydrothermo-chimique présentée, peut être évaluée selon des critères techniques, économiques et environnementaux.

\subsubsection{Aspects techniques}

La faisabilité de la technique hydrothermo-chimique est basée sur sa simplicité et sur la possibilité de produire du chitosane de pureté de grade industriel. La production du chitosane, par cette technique, est simple et s'opère seulement en deux étapes à des températures inférieures au point d'ébullition. Dans les conditions optimales proposées par l'application de la technique, le chitosane obtenu est de bonne qualité (90\% de DD) et peut être utilisé dans le domaine du traitement des eaux usées. Les infrastructures nécessaires reposent sur un appareillage commun et des procédures opératoires simples, accessible à travers le monde. La simplicité de cette nouvelle procédure ne nécessite pas non plus du personnel hautement qualifié.

\subsubsection{Aspects économiques}

Le court temps de réaction et la quantité de réactifs de digestion moins importante, relativement à la méthode conventionnelle, permettent de réaliser des économies tant sur la taille des infrastructures que sur les coûts de production. En raison de la possibilité d'utiliser une température plus basse, la consommation énergétique pour la production du chitosane à $90 \%$ de DD est moins élevée par rapport aux autres techniques chimiques citées dans la littérature $\left(\mathrm{NaOH} 50 \%, 150^{\circ} \mathrm{C}\right)$. En conséquence, une réduction de l'énergie de $36 \%$ et de la quantité de $\mathrm{NaOH}$ de $25 \%$ pourrait être atteinte par rapport à la technique conventionnelle en trois étapes. De plus, le coût d'investissement des équipements de production n'est pas élevé en comparaison à ceux requis pour l'application de la technique enzymatique ou thermo-mécanique. Ces dernières nécessitent non seulement une installation d'appareillage pour ces deux techniques, mais également un nombre important d'équipements annexes (système d'alimentation, autoclaves en cascade et pressurisation sous atmosphère d'azote). Ainsi, l'élimination de ces équipements et les conditions d'opération diminuent le coût de production et donc améliore sa rentabilité.

\subsubsection{Aspects environnementaux}

La technique proposée en deux étapes s'opère dans des conditions thermiques moins élevées qui permettent d'éliminer les taux d'émissions polluantes. Ce fait présente donc une réduction sérieuse des impacts environnementaux nuisibles ainsi que la corrosion et la protection des infrastructures. Les produits chimiques disponibles sont les solutions diluées de $\mathrm{HCl}$ et de $\mathrm{NaOH}$. Aucun solvant organique n'est utilisé par cette technique (tel que diéthylether dans la méthode de Fujita). En outre, l'utilisation d'acides et de bases à des concentrations plus faibles ainsi qu'une température de réaction plus basse sont des facteurs qui diminuent les risques sur la santé et la sécurité au travail. De plus, ces concentrations plus faibles ne compromettent pas la possibilité de recycler ou de réutiliser les acides et les bases, diminuant du même coup l'empreinte écologique du procédé.

\section{CONCLUSION}

La valorisation des exosquelettes de crevettes grises par extraction du chitosane selon la technique hydrothermochimique proposée en deux étapes permet de diminuer le temps de production d'au moins quatre fois par rapport à la technique classique en trois étapes (trois à quatre jours). De plus, la consommation de produits chimiques de digestion et d'énergie est aussi diminuée significativement. Le chitosane obtenu par cette technique en deux étapes est de bonne qualité. En effet, le degré de désacétylation est supérieur à $90 \%$ dans les conditions optimales pour la protéination et la désacétylation simultanée. Cette technique présente l'intérêt d'être plus simple et plus sécuritaire. Elle permettrait de produire un chitosane avec une moins grande empreinte écologique que les techniques publiées jusqu'à présent (CHATTERJEE $e t$ al., 2005; NIEDERHOFER et MÜLLER, 2004).

Les futures recherches devraient donc associer les principes 
d'analyse du cycle de vie des produits et inclure la gestion des sous-produits de la fabrication du chitosane ainsi que les boues résiduelles du traitement des eaux.

\section{REMERCIEMENTS}

Je tiens à remercier tout le personnel de la Station expérimentale des procédés pilotes en environnement de l'École de technologie supérieure (STEPPE-ÉTS) pour m'avoir permis de réaliser les expériences.

\section{RÉFÉRENCES BIBLIOGRAPHIQUES}

AIBA S., M. IZUME, N. MINOURA et Y. FUJIWARA (1985). Studies on chitin - preparation and properties of chitin membranes. Carbohydr. Polym., 5, 285-295.

APHA, AWWA et WEF (1995). Standard methods for examination of water and wastewater. $19^{\text {th }}$ Edition. American Public Health Association, American Water Works Association, Water Environment Federation, Washington, D.C., États-Unis.

BAXTER A., M. DILLON et K.D. ANTHONY TAYLOR (1992). Improved method for I.R. determination of the degree of $\mathrm{N}$-acetylation of chitosan. Int. J. Biol. Macromol., 14, 166-169.

BLAIR H.S., J. GUTHRIE, T. LAW et P. TURKINGTON (1989). Chitosan and modified chitosan membranes I. Preparation and characterisation. J. Appl. Polym. Sci. 33, 641-656.

BRINE C.J., P.A. SANDFORD et J.P. ZIKAKIS (1992). Advances in chitin and chitosan. Elsevier, Londres, Angleterre.

CHANG K.L.B., G. TSAI, J. LEE et W.R. FU (1997). Heterogeneous N-deacetylation of chitin in alkaline solution. Carbohydr. Res., 303, 327-332.

CHATTERJEE S., M. ADHYA, A.K. GUHA et B.P. CHATTERJEE (2005). Chitosan from Mucor rouxii: Production and physico-chemical characterization. Proc. Biochem., 40, 395-400.

CHEN L., D. CHEN et C. WU (2003). A new approach for the floculation mechanism of chitosan. J. Polym. Env., 11, 89-92.
CHEN C.H., F.Y WANG et Z.P. OU (2004). Deacetylation of $\beta$-chitin. I. Influence of the deacetylation conditions. J. Appl. Polym. Sci., 93, 2416-2422.

CUROTTO E. et F. AROS (1993). Quantitative determination of chitosan and the percentage of free amino groups. Anal. Biol., 211, 240-241.

COUGHLIN R.W., M.R. DESHAIES et E.M. DAVIS (1990). Chitosan in crab shell wastes purifies electroplating wastewater. Environ. Prog., 9, 35-40.

DAUTZENBERG H., W. JEAGER, J. KOTZ, B. PHILLIPP, C. SEIDEL et D. STSCHEERBINA (1994). Polyelectrolyte, formation, characterization and application. Hanser, New York, N.Y., États-Unis.

FENTON D.M. et D.E. EVELEIGH (1981). Purification and mode of action of a chitosanase from Penicillium islandicum. J. Gen. Microbiol., 126, 151-165.

FREHAUT G. (2002). Un procédé "propre" de production du chitosane. Bulletin Electronique (BE) Allemagne, 121 p.

GASTON C. (1983). Les réactions chimiques en solution aqueuse et caractérisation des ions. Masson, Paris, France.

GOOSEN M.F.A. (1997). Applications of chitin and chitosan. Technomic, Lancaster, PA, États-Unis.

HIRANO S. (1989). Chitin and chitosan. Elsevier, New York, NY, États-Unis.

HIRANO S. (1996). Chitin biotechnology applications. Biotechnol. Annu. Rev., 2, 237-258.

HIRANO S. (1999). Chitin and chitosan as novel biotechnological. Polym. Int., 48, 732-734.

JUANG R.S. et R.C. SHIAU (2000). Metal removal from aqueous solutions using chitosan-enhanced membrane filtration. J. Membr. Sci., 165, 159-167.

KHAN T.A., K.K. PEH et S.C. HUNG (2002). Reporting degree of deacetylarion values of chitosan: The influence of analytical methods. J. Pharm. Sci., 5, 205-212.

KIM T.Y., K.J. KIM, H. MOON et J.H. YANG (1999). Adsorption of cupric ions on chitosan. J. Korean Ind. Eng. Chem., 10, 268- 274.

METHACANON P., M. PRASITSILP, T. POTHSREE et J. PATTARAARCHACHAI (2003). Heterogeneous 
$\mathrm{N}$-deacetylation of squid chitin in alkaline solution. Carbohydr. Polym., 52, 119-123.

MOORE G.H. et G.A.F. ROBERTS (1980). Determination of the degree of $\mathrm{N}$-acetylation of chitosan. Int. J. Biol. Macromol., 2, 115-116.

MURCOTTE S. et D.R.F. HARLEMAN (1993a). The efficacy of chitosan and other natural polymers in removing COD, TSS, heavy metals and PAHs from municipal wastewater. Sea Grant College Program Massachusetts Institute of Technology, Project No. RT-1, Cambridge, MA, ÉtatsUnis, $71 \mathrm{p}$.

MURCOTTE S. et D.R.F. HARLEMAN (1993b). Mit jar test of Massachusetts reservoir water using the natural polymer chitosan with bentonite. Sea Grant College Program Massachusetts Institute of Technology, Project No. 92-A5, Cambridge, MA, États-Unis, 47 p.

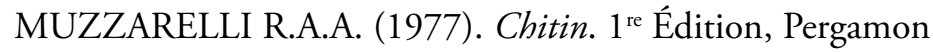
Press, Oxford, Angleterre.

MUZZARELLI R.A.A. et R. ROCHETTI (1985). Determination of degree of acetylation of chitosan by first derivative ultraviolet spectrophotometry. Carbohydr. Polym., 5, 461-472.

MUZZARELLI R.A.A. (1996). Chitosane-based dietary foods. Carbohydr. Polym., 29, 309-316.

NIOLA F. (1991). Étude de la réaction de désacétylation de la chitine par le procédé thermo-mécano-chimique. Mémoire de maîtrise, Université de Sherbrooke, Sherbrooke, QC, Canada, 128 p.

NIEDERHOFER A. et B.W. MÜLLER (2004). A method for direct preparation of chitosan with low molecular weight from fungi. Eur J. Pharm. Biopharm., 57, 101-105.

NO H.K, Y.I. CHO, H.R. KIM et S.P. MEYERS (2000). Effective deacetylation of chitin under conditions of 15 psi $/ 121^{\circ}$ C. J. Agric. Food Chem., 48, 2625-2627.

NWE N. et W.F. STEVENS (2002). Production of fungal chitosane by solid substrate fermentation followed by enzymatic extraction. Biotechnol. Lett., 24, 131-134.

OKAFOR N. (1965). Isolation of chitin from the shell of the cuttle fish, Specia officinalis 1. Mucoproteins and mucopolysaccharides. Biochim. Biophys. Acta, 101, 193-200.
R.P. OVEREND (1990). Chitin/chitosan transformation by thermo-mechano-chemical treatment including characterisation by enzymatic depolymerisation. Biotechnol. Bioeng., 36, 310-315.

PELLETIER A. (1991). Valorisation de la chitine par sa transformation en produits chitine/chitosane variés grâce à des procédés thermo-mécano-chimiques et enzymatiques. Thèse de Doctorat, Université de Sherbrooke, Sherbrooke, QC, Canada, 160 p.

PLONSKI B.A., H.V. LUONG et E.J. BROWN (1990). Arsenic sorption by chitosan and chitin deacetylase production by Mucor ruoxii. Biorecovery, 1, 239-253.

ROBERTS G.A.F. (1992). Chitin chemistry. MACMILLAN PRESS, Londres, Angleterre.

TOKURA S. et N. NISHI (1994). Specification and characterization of chitin and chitosan. Asian-Pacific chitin and chitosan symposium, Bangi, Malaysie, pp. 67-86.

WANG T., M. TURHAN et S. GUNASEKARAN (2004). Selected properties of $\mathrm{pH}$-sensitive, biodegradable chitosanpoly(vinyl alcohol) hydrogel. Polym. Int., 53, 911-918.

WU A.C.M. (1988). Determination of molecular-weight distribution of chitosan by high-performance liquid chromatography. Meth. Enzymol., 161, 447-452. 